\title{
Scaling Blockchains Without Giving up Decentralization and Security
}

\author{
A Solution to the Blockchain Scalability Trilemma
}

\author{
Gianmaria Del Monte \\ Engineering Dept. \\ Roma Tre University \\ Roma, Italy \\ gia.delmonte@stud.uniroma3.it
}

\author{
Diego Pennino \\ Engineering Dept. \\ Roma Tre University \\ Roma, Italy \\ pennino@ing.uniroma3.it \\ 0000-0001-5339-4531
}

\author{
Maurizio Pizzonia \\ Engineering Dept. \\ Roma Tre University \\ Roma, Italy \\ pizzonia@ing.uniroma3.it \\ 0000-0001-8758-3437
}

\begin{abstract}
Public blockchains should be able to scale with respect to the number of nodes and to the transactions workload. The blockchain scalability trilemma has been informally conjectured. This is related to scalability, security and decentralization, stating that any improvement in one of these aspects should negatively impact on at least one of the other twos. In fact, despite the large research and experimental effort, all known approaches turn out to be tradeoffs.

We theoretically describe a new blockchain architecture that scales to arbitrarily high workload provided that a corresponding proportional increment of nodes is provisioned. We show that, under reasonable assumptions, our approach does not require tradeoffs on security or decentralization. To the best of our knowledge, this is the first result that disprove the trilemma considering the scalability of all architectural elements of a blockchain and not only the consensus protocol. While our result is currently only theoretic, we believe that our approach may stimulate significant practical contributions.

Keywords-Blockchain, Distributed Ledger Technology, Sclability, Security, Decentralization, Trilemma.
\end{abstract}

\section{INTRODUCTION}

Scalability of public blockchains is extremely important for their success. All communities and companies engaged in the development of public blockchains strive to create solutions to support a large number of nodes and/or large workloads (in terms of transactions per second). While distributed systems or peer-to-peer systems can scale very well with respect to these parameters, blockchains, till now, have represented a very hard challenge. The difficulty comes from the interplay of contrasting requirements in the blockchain design. Vitalik Buterin, founder of the Ethereum project, summarized his understanding about this problem by introducing the socalled blockchain scalability trilemma. This trilemma states that regarding scalability, security and decentralization, any improvement in one of these aspects negatively impacts on at least one of the other twos.

Intuitively, consider an ideal blockchain, in which nodes are all equal (with same and constant cpu, bandwidth, storage, etc.). If we double both load and number of nodes, the ratio between load and available processing resources is constant, and scalability might, in principle, be possible. The obvious question is if it is possible to design a blockchain in which all activities (consensus, storage, and communication) are distributed (and not just replicated) across nodes, keeping both high security and high decentralization, independently from the load and the number of nodes.

Current blockchains broadcast all pending transactions and accepted blocks to all nodes. Hence, each node is a scalability bottleneck, since its processing power and its bandwidth are bounded. While a large literature addresses the scalability of the consensus algorithm, this is only a part of the story. The sharding approach, propose to partition the whole network (comprising nodes, blocks, consensus, and transaction history) into several smaller networks (shards), with some form of coupling among them (to handle inter-shard transactions). However, shards have less nodes than the whole network, reducing decentralization and security, and inter-shard transactions may pose a scalability challenge.

In this paper, we theoretically describe a novel blockchain architecture for which the maximum workload that can be processed is proportional to the number of nodes involved in the blockchain. Our scalability result is related to all architectural aspects, i.e., no node is ever forced to receive, process or store a quantity of data that is proportional to the whole workload. In our approach, decentralization and security, are not affected when the number of nodes is increased. This derives from the adoption, as a building block, of a randomized committeebased consensus, like the one described in [5].

The value of our contribution is twofold. Firstly, it shows that, under realistic assumptions, it is possible to solve the blockchain scalability trilemma. Secondly, it provides a construction that can be of inspiration for practical realizations.

The rest of the paper is structured as follows. In Section II, we quickly review some related literature. In Section III, we provide basic definitions, assumptions and some background. In Section IV, we focus on scalability problems of current solutions. In Section V, we first present the main ideas of our architecture and then we detail the tasks performed by 
each committee. In Section VI, we formally prove correctness. In Section VII, we show a formal scalability result. In Section VIII, we discuss the effectiveness of our approach and some other aspects. In Section IX, we draw the conclusions.

\section{StATE OF THE ART}

Despite its practical relevance and its potential theoretical impact, the blockchain scalability trilemma [22] has not a formal definition in scientific literature. However, "the trilemma" is often cited in research works. For example, a taxonomy of consensus algorithm based on the scalability trilemma is provided in [1] and surveys on blockchain scalability explicitly cite it [24], [27].

Concerning scalability, the most interesting results are about sharding. However, all of them suffer, in different ways, from the strong partitioning of the whole blockchain. RapidChain [26] requires strong synchronous communication among shards which is hard to achieve. AHL [7] is an approach that turns out to be expensive when a transaction involves multiple shards. Omniledger [13] requires every participant in the consensus protocol (a subset of the nodes in each shard) to broadcast a message to the entire network to verify transactions. It also requires the users to participate actively in cross-shard transactions verification. Elastico [15] and Monoxide [21], require to execute expensive proof-of-work to decide which shard should process a transaction. Other proposals are targeted to scalability of consensus algorithms, like the EOS network, which is analyzed in [25], Algorand [5], and Ouroboros [8], [12].

A fundamental role in blockchain scalability is played by Authenticated Data Structures (ADS) and related techniques. The works [5] and [3] propose to use authenticated data structures to scale with respect to the size of the blockchain state. Other works related to scalability of ADSes are [18], [17], [10], [16], [9].

\section{BASIC DEFINITIONS AND ASSUMPTIONS}

In the following, we introduce some definitions and assumptions that are used in the rest of the paper.

We call candidate (or pending) transactions those that are generated by users but are not (yet) processed by the blockchain. A confirmed (or accepted or committed) transaction is a transaction that was successfully processed by the blockchain. The history of the blockchain is a totally ordered sequence of confirmed transactions. For simplicity, in the rest of the paper, we mostly focus on a simple model of blockchain that realizes a pure cryptocurrency. In other words, each address (or account) is associated to a wallet with a non negative balance and a transaction just moves currency from a wallet to another changing balances, accordingly. While the results of this paper may be applicable in a more powerful model, for simplicity, we do not consider more complex cases. The state of the blockchain is the balances of all addresses, at a certain instant. Since confirmed transactions are totally ordered, the state of the blockchain is defined between two consecutive accepted transactions. The sequence of confirmed transactions is split into blocks that are sequentially numbered. Pending transactions are confirmed when a new block is created (or mined). The mining of a new block requires to (1) select a subset of pending transactions, (2) order them, and (3) verify that certain consensus rules are fulfilled when a transaction is applied to the previous state. In practice, consensus rules may be complex, but, in our model, they are limited to keeping non-negative balances.

The nodes of a blockchain network act as peers in the sense that they perform the same actions. Commonly, they broadcast candidate transactions (even though, in the approach described in this paper this is not true). Each node keeps a set of candidate transactions it knows. Nodes jointly perform a consensus algorithm (or consensus protocol) to reach consensus on the transactions to be included in the next block. This also implies a consensus on the state after that block (i.e., after the last transaction of the block). We assume that consensus does not introduce forks as in [5]. In our approach, the transactions to be included in the next block are decided by a joint work of a number of committees, each of them performing a consensus algorithm. This paper describes in detail how this can be done.

We consider all nodes to have always the same constant amount of resources in terms of CPU, storage, and network bandwidth. For simplicity, we also assume that honest nodes are reliable, that all communications between nodes are instantaneous, and that the backbone of the underlying network does not introduce any bottleneck and is reliable.

The stream of candidate transactions is the workload (or simply load) of the blockchain and its magnitude is a frequency measured in transactions per seconds. In the following, we assume that accounts whose balance is changed by candidate transactions are uniformly distributed on the address space. The time a candidate transaction takes to be confirmed is called (confirmation) latency. The maximum throughput of the blockchain is the frequency of candidate transactions that it is possible to confirm with bounded latency, i.e., avoiding indefinitely growth of the set of pending transactions. When workload is less than the maximum throughput, we say that the blockchain is well-provisioned (for that workload). Since we are interested in investigating scalability of blockchains, we compare situations in which the same blockchain architecture is adopted with different number of nodes and different workload. We say that a blockchain architecture scales when starting from a well-provisioned blockchain, it is possible to give a new well-provisioned blockchain with proportionally increased load and nodes. For simplicity, we do not deal with dynamically changing number of nodes.

In the rest of the paper, we also assume knowledge of Merkle trees. A Merkle tree $T$ is a complete binary tree in which each leaf contains a balance of an address and each internal node contains the cryptographic hash of the content of its children. The root of $T$ contains the root-hash of $T$. From $T$, it is possible to provide a (logarithmic length) proof of the value of each of the leaf $v$ of $T$, by providing the content of the siblings of the nodes on the path from $v$ to the root. The proof can be verified only against the root-hash of 
$T$ from which it is derived and it is hard for an attacker to synthesize a valid proof. If proofs are needed for only a subset $S$ of the leaves of $T$, it is possible to prune $T$ so that size of $T$ is proportional to $|S|$ and it is still possible to obtain proofs for each element of $S$. Further details about the use of pruned Merkle trees in blockchains can be found in [3].

\section{Problems of Current Approaches}

In this section, we list some relevant aspects that make current common public blockchains architecture not scalable (according to the scalability definition given in Section III).

In this paper we focus on the following problems.

1) New candidate transactions are always broadcasted to all (validating/mining) nodes.

2) There exists a set of (validating/mining) nodes (possibly comprising all nodes), each processing all candidate transactions that have to be included in the next block.

3) Each new block is broadcasted to all nodes.

Each of these aspects implies that, to well-provision the blockchain, individual nodes have to increase computing power and bandwidth even under proportionality condition.

We purposely avoid to mention scalability problems related to the computational complexity of the consensus protocol, since these three aspects are independent from it and are relevant even for blockchains that adopt light consensus protocols. We also avoid to mention the problem of storing the whole blockchain state in each node, which is already addressed in other works [3], [14].

In literature, most of the proposals that address the above scalability problems introduce some form of sharding, which is a way to partition the blockchain network and the transactions, in effect, creating a sort of federation of a multiplicity of blockchain networks. The sharding technique suffers of a number of problems. The most hard-to-solve ones derives from the fact that state is partitioned across shards. Hence, if shards are many, most transactions turn out to change the state of more than one shard. These are called inter-shard transactions. Clearly, each transaction should be atomic, which is not so simple to achieve in a sharded environment. Typically this introduces inefficiencies related to inter-shard communication (usually performed using some form of broadcast) and the need of techniques similar to a two-phase commit to ensure that all transactions executions are atomic. Another strong criticism is about security, since smaller shards are supposed to allow for better scalability but are deemed to be less secure than larger ones.

The contribution of this paper is the description of an architecture that aims at addressing the three above-mentioned scalability problems. We do this without relying on sharding, in the sense, in our case, the blockchain is one. However, we introduce a way of dynamically sharing the load among nodes. Our solution intends to apply a parallel version of the Algorand consensus approach [5]. We also leverage the idea of distributing the storage of the state, as described in [3].

\section{A Scalable Blockchain ArChitecture}

In this section, we describe an architecture that achieves scalability, as defined in Section III. We first informally describe ideas that make scalability possible in our architecture, then we list in detail all the components of the architecture and their behavior.

\section{A. Main Ideas}

a) Committees: In our approach, there are a number of committees that collectively work to perform the computation needed to validate and confirm transactions and to compute the new block. Each committee is made of a number of nodes called members. The way members of each committee are selected is not important to understand our architecture. However, for security reasons, a randomized approach that regularly change committee members can be adopted (like for example in [5]). We assume that all committees are equal sized. Their size is fixed and does not change when the number of nodes in the network changes. The committees cooperates by exchanging messages. A discussion about inter-committee communication and of periodically changing the committee members is provided in Section VIII.

b) Blocks: Differently from the common approach, in our architecture, a block conceptually aggregates transactions, but transactions are never explicitly represented in the broadcasted block. In fact, the transactions related to a block are proportional to the workload. Forcing a node to receive all of them would impair scalability. Instead, for each new block, we only broadcast constant size data. We call block this constant size data. Our block can be considered equivalent in content to the block header of other traditional approaches. For our theoretical analysis, it is only relevant to know that the block contains the hash of the previous block, and the hash of the blockchain state after the application of all transactions of the block. The state hash is computed on the basis of a Merkle tree, hence we call it state root-hash. In the following, we explain how the computation of the state root-hash is shared across several committees. In principle, blocks might also contain a hash of the transactions of the block. However, this is not strictly needed for the correctness of the execution, which computes the new state on the basis of the previous one. Hence, we ignore it. Consider also that techniques similar to those that we propose for scaling with respect to the computation the state root-hash could be adopted for a roothash that summarizes the transactions of the block.

c) Storage: In our solution, we are interested in storing the state of the blockchain (a similar approach can be adopted for transaction history, but we do not include it in our model). For scalability reasons, it is not possible for all nodes to store the whole state. This is not only because of the size of the needed storage, but also because processing updates to the whole state would require an amount of resources proportional to the workload. Bernardini et al. [3] and Vault [14] propose approaches that do not require for all nodes to store the whole blockchain state. In these approaches, a node may not even store any state at all and still be able to participate 
in transactions confirmation and block creation activities. In the following, we refer to a node that stores a part of the blockchain state as a storage node. The cited works, consider a (complete and binary) Merkle tree on the whole address space in which each leaf is an address (comprising unused addresses). We denote this Merkle tree by $W$. Storage nodes store only a part of the state (a subset of all accounts) and the corresponding part of $W$, that covers all the paths from stored addresses to the root, pruning the rest of $W$ (see details in [3]). The root-hash of $W$ is the state root-hash. Since blockchain state changes, the block contains the state root-hash of the state after the application of the last transaction of the block.

d) Transaction creation: As in [3] and [14], in our approach, a node $n$ that intends to create a transaction has the responsibility to provide cryptographic proofs of the balances of the accounts that are going to be changed by the transaction (i.e., of the accounts involved in the transaction). These cryptographic proofs are asked by $n$ to one or more storage nodes. Since each storage node stores a pruned Merkle tree, they are able to provide that proof for the accounts they store. However, as will be clear in the following, the balances provided by storage nodes are related to a state that is delayed by a few blocks. The proof $p$ obtained from a storage node is related to a state of the blockchain after a certain block $B$, intending that its is valid with respect to the state root-hash in $B$. We also simply write that $p$ is related to $B$. Since nodes keep only a truncated list of blocks (see below), proofs that are too old cannot be validated and we say that they are expired.

e) Pipelining: Consider the computation needed to validate and confirm transactions and then to compute the new state root-hash to be included in a new block. The effort needed for this task is clearly proportional to the workload. To scale, it is important to distribute this computation over several committees. For this reason, we introduce a pipeline in which the computation is performed in several stages. In each stage, the computation can be further distributed across several committees, using a parallel processing approach. The output of the last stage is a new block.

We suppose that the time is spliced into equal length rounds. Rounds are sequentially numbered. In each round, each committee performs its task for a certain pipeline stage. The result of the computation of a committee is communicated to the committees that, in the next round, need it for the next pipeline stage. For simplicity, we assume that all communications are instantaneous. A discussion on communication problems is provided in Section VIII.

We denote by $B_{i}$ the block produced as output of the last stage in round $i$. We denote the block that contains the transactions that entered the pipeline at round $i$ by $B^{i}$. If the pipeline has $q$ stages, the transactions that enter the pipeline at round $i$, and that are accepted, will be part of the block produced as output by the last stage that runs at round $i+q-1$. Hence, we have that $B^{i}=B_{i+q-1}$. The first round in which $B^{i}$ can be used by any node is round $i+q$. In Figure 2, an example of pipeline with $q=4$ is depicted. Details are explaind in the following.
Each produced block is propagated to all nodes. Each storage node replies only with proofs related to already produced blocks. We assume that a node that creates a transaction $x$ takes one round to retrieve all the proofs for balances of addresses involved in $x$. These proofs are validated in the first stage of the pipeline. Note that, at that time, these proofs are old by at least $q$ rounds.

f) Truncated block history: As in [3], we assume each node does not keep all the blocks, but only the last $d$ blocks received. That is, at round $i$, each node stores blocks $B_{i-1}=B^{i-q}, \ldots, B_{i-d}=B^{i-q-d+1}$ and previous blocks are forgotten. A proof $p$ related to $B_{j}$ is expired at round $i$ if $j<i-d$ (i.e., nodes have forgotten $B_{j}$ needed to validate $p$ ). Since in round $i$ the last available block is $B_{i-1}$, a storage node replies with proofs related to that block. In our model, we assume a node takes one round to create a transaction (asking for proofs to the storage). Hence, for the nodes participating in committees of the first pipeline stage to be able to verify balance proofs, it should be $d \geq 2$. In the following we assume $d=2$. In a practical realization, $d$ might be larger to compensate delays of the network [3].

\section{B. The architecture}

In Figure 1a, we show the proposed architecture and the flow of information within it. We describe it from left to right.

Any node can create a candidate transaction. As described above, a new candidate transaction should come with balances of the involved accounts and with corresponding proofs of integrity, related to a previous round. This can be obtained from a storage node. Candidate transactions are not broadcasted into the network (nothing is broadcasted in our approach but constant size blocks), instead, they are sent to a limited number of nodes as described below.

The validation of the set of transactions that have to be included in a block is performed by Confirmation Committees (CC). We denote each distinct CC by $C_{k}$ with $k=1, \ldots, N_{c}$, where $N_{c}$ is the number of CCes. When relevant, we write $C_{k}^{i}$ intending to denote the $k$-th confirmation committee that runs in the $i$-th round. The node that creates a new transaction $x$ sends it to $C_{k}^{i}$, with $k=\left(\operatorname{hash}\left(x_{\mathrm{src}}\right) \bmod N_{c}\right)$, where $x_{\text {src }}$ is the account whose balance is charged by $x$. We intend that $x$ is received by $C_{k}^{i}$ before the start of round $i$ and hence $C_{k}^{i}$ can process it during round $i$. We say that $C_{k}^{i}$ is responsible for that transaction. The set of candidate transactions for which $C_{k}^{i}$ is responsible is denoted $P\left(C_{k}^{i}\right)$. We denote by $P^{i}=\bigcup_{k} P\left(C_{k}^{i}\right)$ the set of candidate transactions processed by all confirmation committees in round $i$. The result provided by $C_{k}^{i}$ is a sequence of transactions denoted $A_{k}^{i}$, with $A_{k}^{i} \subseteq P\left(C_{k}^{i}\right)$.

A fundamental aspect of the algorithm performed by $C_{k}^{i}$ is to obtain, for each transaction $x$, the balance of $x_{\text {src }}$ related to $B^{i-1}$ to check that $x$ complies with the non-negative balance rule. Since proofs attached to transactions are related to $B_{i-2}=B^{i-q-1}$, those proofs are for balances that are old. In fact, they might have been outdated by transactions accepted in the last $q$ rounds, for which the corresponding block is not yet available. Hence, each $C_{k}^{i}$ should also be 


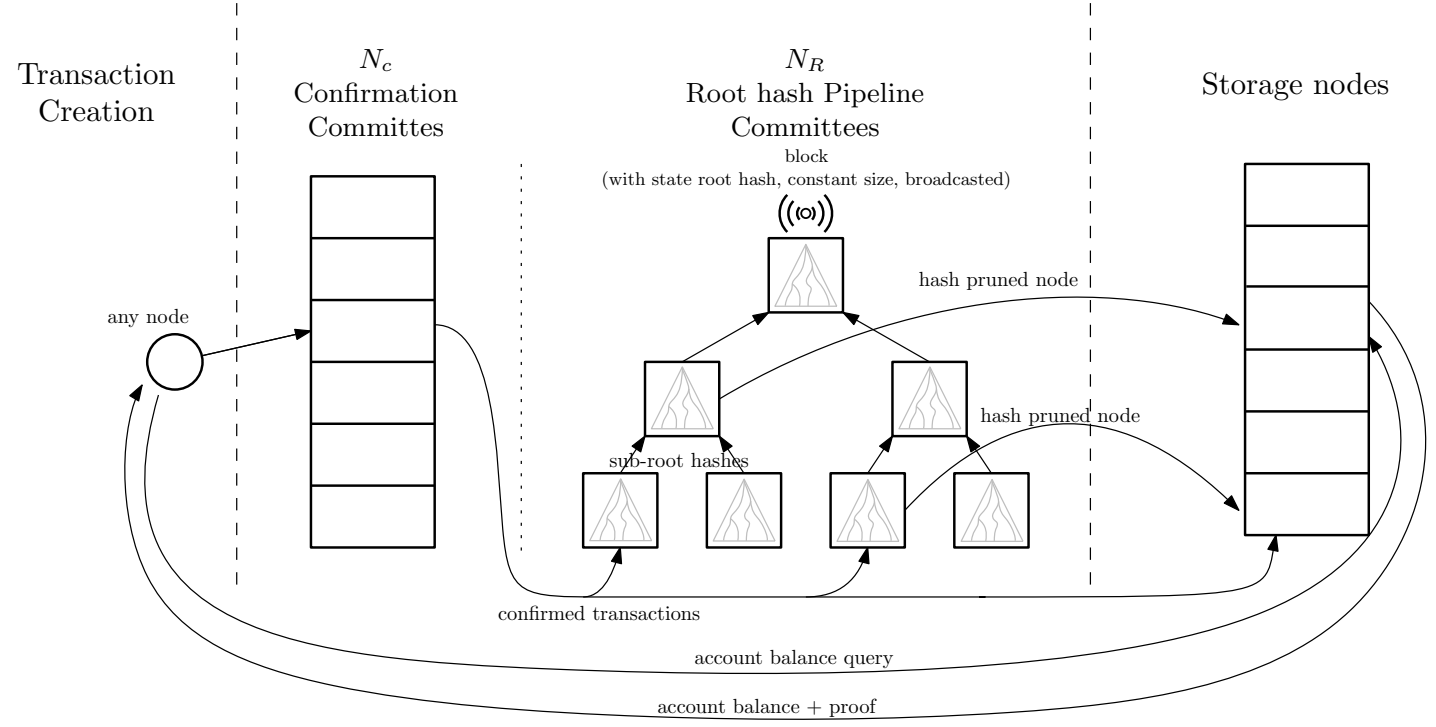

(a)

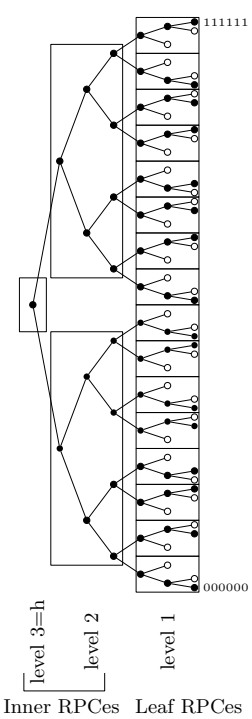

(b)

Fig. 1: In 1a, the flow of data within the proposed architecture. In $1 \mathrm{~b}$, The tree conceptual Merkle tree $W$ partitioned into underlying trees of RPCes. White nodes are pruned.
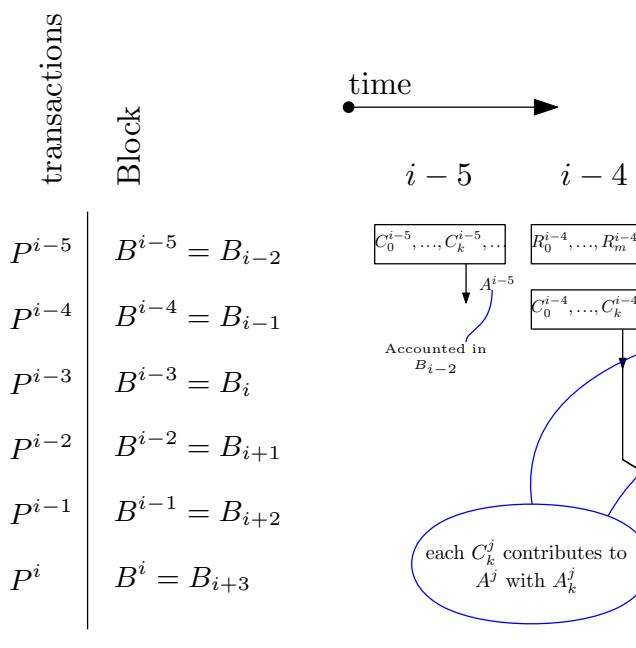

Round Number

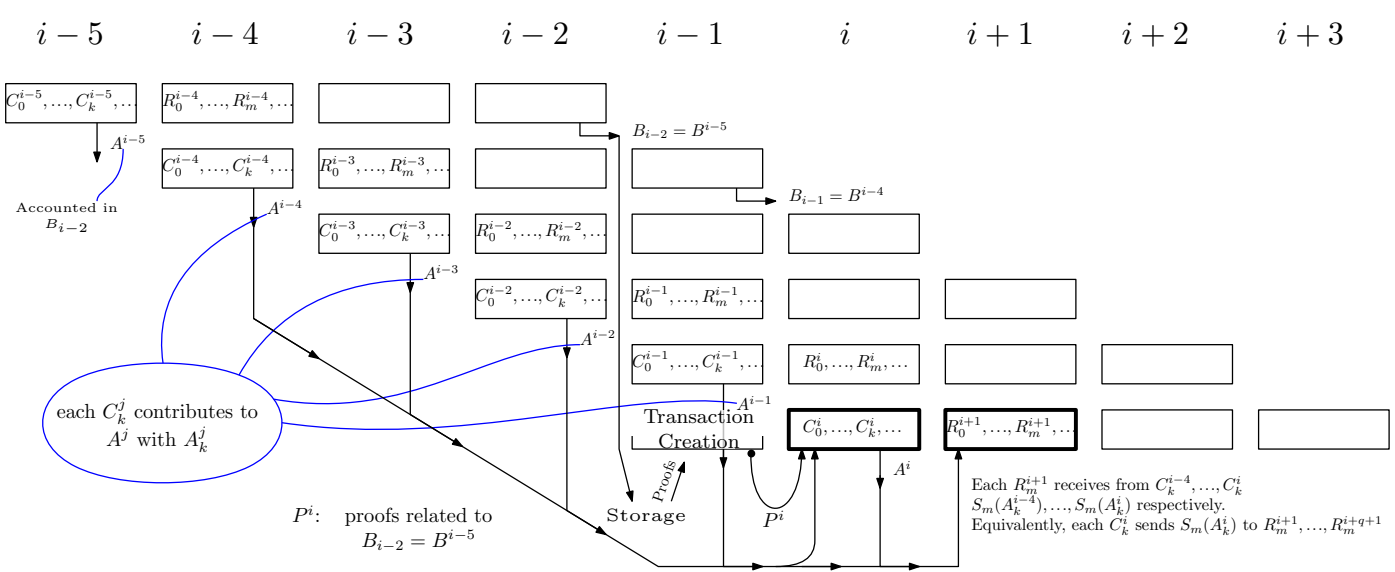

Fig. 2: An example of execution of a pipeline with four stages. In the picture, inputs to $C_{k}^{i}$ and to $R_{m}^{i+1}$ are evidenced.

aware of state changes induced by transactions accepted by $C_{k}^{i-q}, \ldots, C_{k}^{i-1}$, that is, of $A_{k}^{i-q}, \ldots, A_{k}^{i-1}$, respectively (see Figure 2). These transactions are considered to update all account balances involved in $P\left(C_{k}^{i}\right)$ to match state related to $B^{i-1}$. We call time-updating this process. In Figure 2, we depicted the pipeline and put in evidence the inputs for a generic $C_{k}^{i}$.

In our model, each $C_{k}^{i}$ performs the following algorithm (by a suitable consensus protocol).

\section{Algorithm 1 (Confirmation).}

1) It checks that each transaction in $P\left(C_{k}^{i}\right)$ fulfills syntactic rules and proofs are not expired. It discards non- compliant transactions, resulting in $P^{\prime}\left(C_{k}^{i}\right) \subseteq P\left(C_{k}^{i}\right)$

2) It selects an arbitrary order $\bar{T}$ for $P^{\prime}\left(C_{k}^{i}\right)$.

3) Let $\tilde{T}$ be the concatenation of $A_{k}^{i-q}, \ldots, A_{k}^{i-1}$. For each account that appears as source in transactions of $\bar{T}$, consider the last balance from $\tilde{T}$ and from the balances provided by the proofs of transactions in $\bar{T}$.

4) It executes $\bar{T}$ and checks that the resulting balance of each transaction fulfills the non-negative balance rule. Transactions the do not fulfill this rule are discarded. The resulting $A_{k}^{i}$ is derived from $\bar{T}$ where discarded transactions are omitted.

Transactions in $A_{k}^{i}$ should be considered confirmed (or accepted) in the sense they have passed all checks to be 
inserted in $B^{i}$. To allow the confirmation committees of subsequent rounds to perform time-updating, $A_{k}^{i}$ is made available to $C_{k}^{i+1}, \ldots, C_{k}^{i+q}$ and also to other committees, as explained in the following.

The sequence of accepted transactions for that round is denoted $A^{i}=\bigcup_{k} A_{k}^{i}$, where $A^{i}$ is an arbitrary sequence that respects the order of each $A_{k}^{i}$.

Even if $B^{i}$ is yet to be computed, storage nodes can receive from $C_{k}^{i}$ state changes that will be part of $B^{i}$. Transactions in $A_{k}^{i}$ are selectively sent to the storage nodes that need it, to update the part of the state they manage.

The actual computation of $B^{i}=B_{i+q-1}$ requires to compute its state root-hash, which means computing all the hashes of the conceptual Merkle tree $W$ related to the whole state space. This is performed by $N_{R}$ committees, called Roothash Pipeline Committees (RPCes). Each RPC is associated to a part of $W$ as shown in Figure $1 \mathrm{~b}$, called the underlying tree of the RPC. Each underlying tree is rooted to a node whose hash is named sub-root-hash. RPCes themselves form a tree denoted by $W_{\mathrm{RPC}}$, whose internal nodes have $2^{k}$ children, with the exception of the root that have at most $2^{k}$ children. Dimensioning of $k$, and other parameters, is described in Section VII. Upon state changes, each RPC is responsible to compute all hashes for its underlying tree. There are two kinds of RPCes. Leaf RPCes, that are the leaves of $W_{\mathrm{RPC}}$, and inner RPCes, that are all other RPCes. Each leaf RPC is responsible for the interval of contiguous addresses that are leaves of its underlying tree. Since most addresses are unused, leaf RPCes consider a pruned version of the underlying tree containing only the paths from used addresses to its root. The underlying tree of each inner RPC is a complete binary tree (see Section VII). RPCes are partitioned in levels numbered from 1 to $h$. Level 1 contains all leaf RPCes. Level $h$ contains only the root of $W_{\mathrm{RPC}}$. Each level is one stage of the pipeline. Hence, the total number of stages of the pipeline (comprising CCes) is $q=h+1$. Each RPC at level $i<h$ computes a sub-root-hash that is fed as input to its parent in $W_{\mathrm{RPC}}$. The root of $W_{\text {RPC }}$ outputs and broadcasts the new block with the corresponding state root-hash. Theorem 2 of Section VII states that it is possible to dimension $W_{\mathrm{RPC}}$ and the underlying trees of leaf and inner RPCes to ensure scalability.

We write $R_{m}^{i+1}$ to denote a generic leaf RPC that runs in the $(i+1)$-th round and is responsible for the $m$-th portion of the addresses space. As mentioned above, leaf RPCes constitutes the second stage of our pipeline and have to receive as input $A^{i}$, which is the output of the first stage. However, a leaf RPC does not need the whole $A^{i}$. More in detail, each leaf RPC $R_{m}^{i+1}$ receives all and only the transactions in $A^{i}$ that modify the balance of an address for which $R_{m}^{i+1}$ is responsible. In our cryptocurrency model, a single confirmed transaction is sent to two leaf RPCes. If $x$ is a transaction in $A_{k}^{i}, C_{k}^{i}$ sends $x$ to $R_{m}^{i+1}$ only if the source or the destination of $x$ is an address for which $R_{m}^{i+1}$ is responsible. We denote with $S_{m}\left(A^{i}\right) \subseteq A^{i}$ the set of transactions in $A^{i}$ that have one of its involved addresses in the $m$-th portion of the address space and have to be received by $R_{m}^{i+1}$.
The task performed by $R_{m}^{i+1}$ is to calculate the sub-roothash of its underlying tree related to $B^{i}$. To do this, it needs the status of its underlying tree related to $B^{i-1}$. Since, proofs attached to transactions in $S_{m}\left(A^{i}\right)$ are related to $B_{i-2}=$ $B^{i-q-1}$, they cannot be used alone to compute all hashes of the underlying tree related to $B^{i-1}$. In fact, they might have been outdated by transactions accepted in $A^{i-q}, \ldots, A^{i-1}$ for which the corresponding block is not yet available. Hence, each $R_{m}^{i+1}$ should also be aware of $S_{m}\left(A^{i-q}\right), \ldots, S_{m}\left(A^{i-1}\right)$. Each $R_{m}^{i+1}$ considers the proofs of these transactions according to their order to calculate all hashes of the pruned underlying tree related to $B^{i-1}$. We call time-shifting this process (a similar process is described in [3]). To allow the leaf RPCes of subsequent rounds to perform time-shifting, each $C_{k}^{i}$ sends $S_{m}\left(A_{k}^{i}\right)$ to $R_{m}^{i+1}, \ldots, R_{m}^{i+q+1}$, as well.

\section{CORRECTNESS}

In this section, we formally prove the correctness of the architecture introduced in Section V.

The following lemma state the correctness of Algorithm 1 when run on only one committee.

Lemma 1 (Correctness of the confirmation algorithm). Algorithm 1 never returns a sequence that entails a violation of the non-negative balance rule.

Proof. By construction of the result in Step 4 of Algorithm 1.

Theorem 1 (Correctness). Given a set of transactions $P^{i}$ processed, at round $i$, by confirmation committees $C_{k}^{i}$ producing accepted transactions sequences $A_{k}^{i}$, the following statements are true.

1) In any sequence $A^{i}=\bigcup_{k} A_{k}^{i}$ such that $A^{i}$ respects the order of each $A_{k}^{i}$, the non-negative balance rule is respected.

2) The state root-hash of $B^{i}=B_{i+q-1}$ is the root-hash of the new state after the application of $A^{i}$.

3) Storage nodes knows the proofs of the addresses they store.

Proof. Concerning Statement 1, observe that $A_{k}^{i}$ satisfy the non-negative balance rule (Lemma 1) and their order is preserved by hypothesis in $A^{i}$. Since for each $k$ the addresses charged in $A_{k}^{i}$ are not charged in any $A_{j}^{i}$ with $j \neq k$, the statement follows.

Concerning Statement 2, note that each leaf RPC $R_{m}^{i+1}$ considers all the transactions that involve addresses for which $R_{m}^{i+1}$ is responsible that are present in sequences $S_{m}\left(A^{i-q}\right), \ldots, S_{m}\left(A^{i-1}\right)$, respecting their order. RPC $R_{m}^{i+1}$ can correctly compute its sub-root-hash to pass to its parent RPC. In fact, if an internal node of its underlying tree is involved in a transaction, $R_{m}^{i+1}$ receives the proofs attached with the transaction. If an internal node of its underlying tree is not involved in any transaction either it is pruned or it is a root of a pruned tree. In the first case, $R_{m}^{i+1}$ does not need it. In the latter case, $R_{m}^{i+1}$ receives its hash in one of the proofs available to it. Since, internal RPCes always receive, form their children, 
the hash values for all the leaves of their underlying tree, computing their sub-root-hash is trivial. Hence, the statement follows.

Concerning Statement 3, note that RPCes compute the roothash on the basis of a pruned version $W^{\prime}$ of $W$, where leaves of $W$ kept in $W^{\prime}$ are all used addresses $U$. Each storage node $n$ stores a pruned version $W_{n}$ of $W$, where leaves of $W_{n}$ are all addresses $U_{n}$ that $n$ intends to store. Since $U_{n} \subseteq U$, also $W_{n} \subseteq W^{\prime}$. Hence, all sub-root-hash of pruned subtrees in $W_{n}$ are known to one of the RPCes, which can communicate it to $n$.

\section{SCALABILITY}

In this section, we formally show the scalability of our approach. For real systems, the workload is usually characterized probabilistically. For simplicity, we reason assuming a deterministic workload. Indeed, similar arguments hold when reasoning with expected values. We also assume balance changes in a round are uniformly distributed across the whole address space. We start by introducing some assumptions and notation.

We denote by $f$ the frequency of transactions of the blockchain workload. We denote by $\Delta$ the duration of a round. We denote by $m=2 f \Delta$ the number of addresses whose balance changes in each round, assuming transactions involve distinct addresses. Let $\tilde{W}$ a pruned version of $W$ where only $m$ leaves are kept, i.e., those related to the addresses that change balance in one round. We note that there is a level $l$ of $\tilde{W}$, above which $\tilde{W}$ is a complete binary tree. As $m$ grows, the pruned part get smaller and $l$ get closer to the leaves.

We denote by $j$ the maximum number of hashes that an RPC can compute in a round. Note that $j$ is constant, since it depends on the CPU power of committees members. We denote by $e$ the maximum number of balance changes that a leaf RPC $R$ can process in one round. Clearly $e$ depends on $j$ and on how changes are distributed in the address space for which $R$ is responsible, since this determines the number of nodes of the pruned underlying tree that $R$ has to deal with. However, by the uniform distribution assumption, $e$ is the same for all leaf RPCes. For simplicity, we assume $j$ to be large enough so that the root of the underlying tree of leaf RPCes is above level $l$. Hence, the underlying tree $U$ of an inner RPC $R$ is a complete binary tree, as stated in Section V-B. Let $k$ be the number of the levels of $U$. The nodes of $U$ are $2^{k}-1$. The children RPCes of $R$ are $2^{k-1}$. For each round, the inner RPC has to compute one hash for each node of $U$. The maximum number of nodes in the underlying tree of an inner RPC is $\hat{j}=2^{\hat{k}}-1$, where $\hat{k}$ is the largest possible integer such that $\hat{j} \leq j$, or equivalently $\hat{k}=\left\lfloor\log _{2}(j+1)\right\rfloor$. The maximum number of levels of the underlying tree of an RPC is also $\hat{k}$. We denote by $S(N, f)$ a blockchain system $S$, with the architecture described in Section $\mathrm{V}$, with $N$ nodes and with a workload at frequency $f$.

Lemma 2. Consider a blockchain system $S(N, f)$, of $N$ nodes with workload $f$. Let $e$ be the maximum number of balance changes that a leaf RPC can handle per round, and $\hat{j}$ be the maximum number of hashes an inner RPC can compute per round. If $S$ is well dimensioned, the number of leaf RPCes is at least $2^{\left\lceil\log _{2}(m / e)\right\rceil}$ and the number of inner RPCes is at least $\left\lceil\frac{2^{\left\lceil\log _{2}(m / e)\right\rceil}-1}{\hat{j}}\right]$, where $m=2 f \Delta$ and $\Delta$ is the round duration.

Proof. Leaf RPCes should at least be $\lceil\mathrm{m} / \mathrm{e}\rceil$, however, since they have to be the leaves of a complete binary tree, underlying to inner RPCes, their number have to be a power of 2 . Hence, we they have to be $2^{g}$ with $g=\left\lceil\log _{2}(m / e)\right\rceil$. Consider the union of the underlying graphs of all inner RPCes $W_{I}$, its number of levels is $g$, as well.

As $m$ increases, the resource usage of each leaf RPC increases. When resources of leaf RPCes are fully used, their number is doubled. Note that, doublings occur when $m / e$ is a power of 2. Right after a doubling, their resources are half used. Increasing $m$, their resource usage goes from half of its processing capacity to maximum capacity, which occurs right before a doubling.

When leaf RPCes (at level 1 of $W_{R P C}$ ) are doubled, also inner RPCes at level 2 of $W_{R P C}$ are doubled. All inner RPCes have their underlying tree with the maximum number of levels, except for the root of $W_{R P C}$ (see Figure $1 \mathrm{~b}$ ), for which its number of levels is increased by one at each doubling. This occurs until the levels of the underlying tree of the root of $W_{R P C}$ reaches $\hat{k}$. After that, $g$ increases by one and a new root with only one node as underlying tree is added at the top. Since, leaf RPCes are $2^{g}$, the nodes of $W_{I}$ are $2^{g}-1$. Hence, the number of inner RPCes is given by $\left[\left(2^{g}-1\right) / \hat{j}\right]$. Note that both numerator and denominator represent the size of a complete binary tree, with $g$ and $\hat{k}$ levels, respectively. The integer part of the result of the division is the number of inner RPCes with full-sized underlying tree. The reminder is the size of the underlying tree of the root of $W_{R P C}$, which is not full-sized, in general.

The following theorem states the scalability of our approach when nodes and workload are proportionally increased.

Theorem 2 (Scalability). There exists a well-provisioned blockchain system $S(N, f)$, with $N$ nodes and workload frequency $f$, such that, for all $\alpha>1$ such that $\alpha N$ is integer, it is possible to provide a well-provisioned blockchain system $\bar{S}(\alpha N, \alpha f)$.

The above statement holds under the assumption that the balance changes induced by the workload are uniformly distributed across the address space.

Proof. We choose as $S(N, f)$ a well-provisioned system, right after a doubling, i.e., with minimum resource usage for the leaf RPCes. In our setting, committees of $S$ and $\bar{S}$ have the same processing capabilities. We want to prove that $\alpha N$ nodes in $\bar{S}$ are enough for the number of committees needed to process a workload $\alpha f$. We first derive the needed number of CCes in $\bar{S}$ compared to that of $S$ and then we do the same for RPCes. 
Then, we show that they are compatible with the increment of the nodes.

A workload at frequency $f$, generates $f \Delta$ transactions per round. Let $N_{C}$ be the number of CCes in $S$. Since $S$ is wellprovisioned, each $\mathrm{CC}$ is able to process $f \Delta / N_{C}$ transactions per round. The load of $\bar{S}$ is $\alpha f$, hence, with $\alpha N_{C}$ CCes, we obtain in $\bar{S}$ the same resource usage of each CC as in $S$.

Note that, the fact that each $C C$ have to re-process transactions accepted in a constant number of previous rounds does not impact this reasoning. This is true also for the following argument about RPCes.

We use symbols, $m, e, \hat{j}$, and $g$ with the same meaning as before. To simplify the proof, in accordance with Lemma 2, we choose to provision $S$ and $\bar{S}$ with $\left[\frac{2^{\left\lceil\log _{2}(m / e)\right\rceil}}{\hat{j}}\right]$ inner RPCes, possibly leaving one of the inner RPCes without workload. Note that, $S$ is well-provisioned and with minimum resource usage of leaf RPC, i.e. with $f$ right after a doubling. Hence, in our case, $m / e$ is a power of two, $g=\log _{2}(m / e)+1$, and by Lemma 2 and the above choice for the number of inner RPCes, the total number of RPCes in $S$ is $N_{R}=2^{g}+\left\lceil 2^{g} / \hat{j}\right\rceil=$ $2 m / e+[2 m /(e \hat{j})]$. We now consider the total number $\bar{N}_{R}$ of RPCes needed by $\bar{S}(\alpha N, \alpha f)$. For $\alpha=2^{t}$ with $t$ positive integer, it should be $\bar{N}_{R}=2 \alpha m / e+\lceil 2 \alpha m /(e \hat{j})\rceil$. Since, $[2 \alpha m /(e \hat{j})] \leq \alpha[2 m /(e \hat{j})]$, we have that $\bar{N}_{R} \leq \alpha N_{R}$. Hence, $\alpha N$ nodes are enough for $\bar{S}$ to be well-provisioned. Our assumptions imply that $\bar{S}(\alpha N, \alpha f)$ is again right after a doubling and with only half of the resources of leaf RPCes used. Hence, with the same $\bar{N}_{R}, \bar{S}$ is well provisioned till the next doubling, which covers all the values of $\alpha$ such that, $2^{t}<\alpha<2^{t+1}$.

Hence, the needed increment of the number of CCes and of the number of RPCs from $S$ to $\bar{S}$ is at most by a factor of $\alpha$, and $\bar{S}$ has $\alpha N$ nodes. This proves that $\bar{S}$ is well-provisioned regarding processing aspects.

Additionally, we note that the messages sent and received by each committee in $\bar{S}$ in each round is no more that the double of the number of messages sent and received in $S$. Further, we note that the storage can be realized so that the number of storage nodes that store a certain account is bounded.

The above observations complete the proof of the statement.

\section{DISCUSSION AND FUTURE WORKS}

In this section, we discuss the effectiveness or our approach and certain aspects that are not analyzed in the rest of the paper. In particular, it is important to understand if the proposed approach is a better solution to the blockchain trilemma than the previously known ones. This means understanding if scaling requires to limit security and/or decentralization, since the scalability of our appraoch has been formally stated in Section VII.

a) Decentralization: Concerning decentralization, note that in our system all nodes cooperate in the creation of a new block. Further, even if the committees do not have all the same role, we can assign nodes randomly to each committee, possibly changing them periodically (like, for example, in [5]). In this way, the role of the nodes is statistically homogeneous. Due to these considerations, we think that scaling in our approach does not affect decentralization.

b) Security: Considering security, many other research works and practical systems relay on the security of a consensus algorithm run by a restricted set of nodes forming a committee. In this setting, many attacks require the attacker to control the majority of the committee members. However, when committees are randomly selected, this becomes harder as the number of nodes increases. In conjunction with a proofof-stake approach (for example) this protects against Sybil attacks. If consensus algorithm is robust enough, the presence of several committees has a negligible impact on security. In this sense, security of our approach increases when scaling to a higher number of nodes. Clearly, security is about many other aspects, but the difficulty to subvert the consensus is usually considered in the context of discussions about the blockchain trilemma.

It is worth to note that for the correctness of our approach nodes need to keep only a constant amount of blocks. However, for security reasons, nodes can keep more blocks (or other related information). For example, in Vault [14] the join of a new node is securely performed without downloading the whole blockchain. Analogous approaches can be adopted in our context.

A security analysis with respect to a formally stated threat model is leaved as future work.

c) Committee members selection: Our approach is applicable independently from the way members of each committee are selected. Their selection can be done using a public shared source of randomness or using verifiable random functions, as in [5]. However, there is a caveat regarding this in our approach. Since intermediate results of the pipeline are passed to committees that need them in the next rounds, if members of committees change, these have to be decided and published before data is sent to them. Note that, resorting to broadcast is not possible since this would impair scalability.

d) Inter-committee communication: In Section V, we often relayed on the possibility for a committee to communicate data to other committees that need them in the next rounds. Inter-committee communications should be part of the consensus protocol, in the sense that each receiver should accept a message $m$ from a sender committee $S$ only after having checked that $m$ was sent by a number of members above a certain threshold.

e) Network communications: In our architecture, most messages are sent from nodes to a bounded number of other nodes (members of a certain committee or storage nodes storing a certain account). We note that, using unicast communications, the number of actual messages turns out to be quadratic in the size of the committees. While practically this might be a problem, from a theoretical point of view this is not the case, since the size of committees is constant. However, a critical aspect is that unicast communications require the 
destination to be known, which is not easy to obtain in a scalable manner when the committees change regularly.

We think that a multicast approach can be used to fulfill our needs. The formal statement of the requirements of this underlying multicast layer as well as its design is leaved as future work. However, it may be worth to mention that the use of standard multicast techniques may not completely suite our needs. In particular, the following aspects should be considered when adopting multicast for inter-committee communications or for submission of new transactions to the proper confirmation committee.

1) The members of a multicast group might change rapidly, depending on the round duration.

2) The preparation of the multicast groups can be performed in advance with respect to when they are needed, even if this requires to anticipate the disclosure of committee members.

3) The multicast groups are needed for only one round and then discarded. This might simplify the development of a specific technique for this application.

Regarding the use of multicast for messages destined to storage nodes, we note that the number of needed multicast channels might very large: one for each used address.

Many research works about scalable multicast routing are available in literature (see, for example, [20], [11], [23], [19], [2], [4]). Fast-join multicast routing was studied in [6].

f) Synchronization and committee decision failing: In our description, we essentially assumed a sort of global synchronization. In practice, synchronization spread across a large number of nodes (although partitioned in committees) might be difficult to achieve. The problem of modifying our approach to relax synchronization requirements is left as a future work. A related problem is the failure of a committee to reach an agreement, which is unlikely to for a direct attack, but may occur under large network faults. Again, we left the investigation of these aspect as a future work.

\section{CONCLUSIONS}

We showed a novel blockchain design that distributes the burden to create the next block on many parallel executing committees (involving "almost" all nodes) and that avoids broadcast in all cases that are critical for scalability. We provided formal proof of the scalability of our approach and of its correctness. We also discussed how scaling does not impair decentralization and security. Hence, our architecture can be regarded as a solution to the blockchain scalability trilemma, in the studied setting.

Some future works were already mentioned in Section VIII. In particular, from a theoretical point of view, a formal security proof is needed, as well as a further investigation of synchronization and behavior under committee consensus failure. Further, since we assumed to have a scalable multicast protocol, it should be interesting to understand how to realize this protocol and how it is possible to rely on current technology to realize an efficient one. From a practical point of view, an experimentation or simulation with realistic parameters would be desirable.

\section{ACKNOWLEDGMENTS}

We are extremely grateful to Ciro Oliviero for his important contribution in the beginning of this research.

\section{REFERENCES}

[1] Amani Altarawneh, Tom Herschberg, Sai Medury, Farah Kandah, and Anthony Skjellum. Buterin's scalability trilemma viewed through a statechange-based classification for common consensus algorithms. In 2020 10th Annual Computing and Communication Workshop and Conference (CCWC), pages 0727-0736. IEEE, 2020.

[2] Rajesh I Balay, Girish Bhat, Gregory Lockwood, and Rama Krishnan Nagarajan. Scalable ip-services enabled multicast forwarding with efficient resource utilization, July 3 2012. US Patent 8,213,347.

[3] Matteo Bernardini, Diego Pennino, and Maurizio Pizzonia. Blockchains meet distributed hash tables: Decoupling validation from state storage. In Paolo Mori, Massimo Bartoletti, and Stefano Bistarelli, editors, Distributed Ledger Technology Workshop (DLT 2019), volume 2334, pages 43-55, 2019.

[4] Andrei Broder and Michael Mitzenmacher. Network applications of bloom filters: A survey. Internet mathematics, 1(4):485-509, 2004.

[5] Jing Chen and Silvio Micali. Algorand: A secure and efficient distributed ledger. Theoretical Computer Science, 2019.

[6] Sungyean Cho and Myong-Soon Park. Fjm: fast join mechanism for overlay multicast. In Proceedings of 2003 IEEE Conference on Control Applications, 2003. CCA 2003., volume 2, pages 1333-1338. IEEE 2003.

[7] Hung Dang, Tien Tuan Anh Dinh, Dumitrel Loghin, Ee-Chien Chang, Qian Lin, and Beng Chin Ooi. Towards scaling blockchain systems via sharding. In Proceedings of the 2019 International Conference on Management of Data, pages 123-140. ACM, 2019.

[8] Bernardo David, Peter Gaži, Aggelos Kiayias, and Alexander Russell. Ouroboros praos: An adaptively-secure, semi-synchronous proof-ofstake blockchain. In Annual International Conference on the Theory and Applications of Cryptographic Techniques, pages 66-98. Springer, 2018.

[9] Federico Griscioli and Maurizio Pizzonia. Securing promiscuous use of untrusted usb thumb drives in industrial control systems. In Proceedings of the 14th Annual Conference on Privacy Security and Trust (PST 2016), pages 477-484, 2016.

[10] Federico Griscioli, Maurizio Pizzonia, and Marco Sacchetti. Usbcheckin: Preventing badusb attacks by forcing human-device interaction. In Proceedings of the 14th Annual Conference on Privacy Security and Trust (PST 2016), pages 493-496, 2016.

[11] Björn Grönvall. Scalable multicast forwarding. Computer communication review, 32(1):68, 2002

[12] Aggelos Kiayias, Alexander Russell, Bernardo David, and Roman Oliynykov. Ouroboros: A provably secure proof-of-stake blockchain protocol. In Annual International Cryptology Conference, pages 357388. Springer, 2017.

[13] Eleftherios Kokoris-Kogias, Philipp Jovanovic, Linus Gasser, Nicolas Gailly, Ewa Syta, and Bryan Ford. Omniledger: A secure, scale-out, decentralized ledger via sharding. In 2018 IEEE Symposium on Security and Privacy (SP), pages 583-598. IEEE, 2018.

[14] Derek Leung, Adam Suhl, Yossi Gilad, and Nickolai Zeldovich. Vault: Fast bootstrapping for the algorand cryptocurrency. In NDSS, 2019.

[15] Loi Luu, Viswesh Narayanan, Kunal Baweja, Chaodong Zheng, Seth Gilbert, and Prateek Saxena. Scp: A computationally-scalable byzantine consensus protocol for blockchains. See https://www. weusecoins. com/assets/pdf/library/SCP, 20(20):2016, 2015.

[16] E. Etchevès Miciolino, D. Di Noto, F. Griscioli, M. Pizzonia, J. Kippe X. Clotet, G. Leòn, F.B. Kassim, D. Lund, and E. Costante. Preemptive: an integrated approach to intrusion detection and prevention in industrial control systems. International Journal of Critical Infrastructures (IJCIS), 13(2/3):206-236, 2017.

[17] D. Pennino, M. Pizzonia, and A. Papi. Overlay indexes: Efficiently supporting aggregate range queries and authenticated data structures in off-the-shelf databases. IEEE Access, 7:175642-175670, 2019. 
[18] Diego Pennino, Maurizio Pizzonia, and Federico Griscioli. Pipelineintegrity: Scaling the use of authenticated data structures up to the cloud. Future Generation Computer Systems, 2019.

[19] János Tapolcai, József Bíró, Péter Babarczi, András Gulyás, Zalán Heszberger, and Dirk Trossen. Optimal false-positive-free bloom filter design for scalable multicast forwarding. IEEE/ACM Transactions on Networking, 23(6): 1832-1845, 2014.

[20] Jining Tian and Gerald Neufeld. Forwarding state reduction for sparse mode multicast communication. In Proceedings. IEEE INFOCOM'98, the Conference on Computer Communications. Seventeenth Annual Joint Conference of the IEEE Computer and Communications Societies. Gateway to the 21st Century (Cat. No. 98, volume 2, pages 711-719. IEEE, 1998.

[21] Jiaping Wang and Hao Wang. Monoxide: Scale out blockchains with asynchronous consensus zones. In 16th $\{$ USENIX $\}$ Symposium on Networked Systems Design and Implementation ( $\{N S D I\} 19)$, pages 95 112, 2019.

[22] Ethereum wiki project. On sharding blockchains. https://github.com/ ethereum/wiki/wiki/Sharding-FAQ [Accessed 2020-05-25].

[23] Tina Wong and Randy Katz. An analysis of multicast forwarding state scalability. In Proceedings 2000 International Conference on Network Protocols, pages 105-115. IEEE, 2000.

[24] Junfeng Xie, F Richard Yu, Tao Huang, Renchao Xie, Jiang Liu, and Yunjie Liu. A survey on the scalability of blockchain systems. IEEE Network, 33(5):166-173, 2019.

[25] Brent Xu, Dhruv Luthra, Zak Cole, and Nate Blakely. Eos: An architectural, performance, and economic analysis, 2018.

[26] Mahdi Zamani, Mahnush Movahedi, and Mariana Raykova. Rapidchain: Scaling blockchain via full sharding. In Proceedings of the 2018 ACM SIGSAC Conference on Computer and Communications Security, pages 931-948. ACM, 2018.

[27] Qiheng Zhou, Huawei Huang, Zibin Zheng, and Jing Bian. Solutions to scalability of blockchain: A survey. IEEE Access, 8:16440-16455, 2020. 